\title{
Article
}

\section{L’agriculture à Antananarivo (Madagascar) : une approche interdisciplinaire}

\author{
Christine Aubry ${ }^{1}$, Josélyne Ramamonjisoa ${ }^{2}$, Marie-Hélène Dabat ${ }^{3}$, Jacqueline Rakotoarisoa ${ }^{4}$ \\ Josette Rakotondraibe ${ }^{5}$, Lilia Rabeharisoa ${ }^{6}$ \\ 1 Agronome, INRA, UMRSADAPT, 16 rue Claude Bernard, 75231 Paris cedex 05, France \\ 2 Géographe, Professeur à l'Université d'Antananarivo, Faculté des lettres et sciences humaines, BP 907, 101 Antananarivo, \\ Madagascar \\ 3 Économiste, CIRAD, UPRSCRID, BP 853, Ampandrianomby, 101 Antananarivo, Madagascar \\ 4 Agronome, FOFIFA, Département des Recherches rizicoles, BP 1690, 101 Antananarivo, Madagascar \\ ${ }^{5}$ Chimiste, Professeur à l'Université d'Antananarivo, Faculté des sciences, et C3EDM, 101 Antananarivo, Madagascar \\ 6 Agronome, Professeur à l'École supérieure des sciences agronomiques, LRI, BP 3383, 101 Antananarivo, Madagascar
}

\begin{abstract}
Répondre à des appels d'offre en empruntant la voie interdisciplinaire est partie intégrante d'une démarche de recherche qui donne lieu rarement à publication. Le travail qui consiste, pour des chercheurs de disciplines différentes, à se saisir d'un problème pour en faire un objet de recherche partagé est ignoré sur le plan scientifique. Cet article rend compte d'un tel travail préalable où les disciplines confrontent leurs postures de recherche. Il nous apprend tout autant de l'agriculture péri-urbaine à Madagascar que des enjeux scientifiques qui traversent la construction d'un programme de recherche interdisciplinaire.
\end{abstract}

La Rédaction

\section{Mots-clés :}

agriculture urbaine ; multifonctionnalité ; interdisciplinarité ; Madagascar

\section{Keywords:}

urban agriculture; interdisciplinarity; multifunctionality; Madagascar

\begin{abstract}
Résumé - La durabilité de l'agriculture urbaine d'Antananarivo (Madagascar) a été questionnée dans le cadre d'un projet de recherche pluridisciplinaire à visée opérationnelle. Six thèmes de recherche articulent quatre disciplines (agronomie, économie, géographie, chimie) autour de sites, de systèmes de production ou de produits communs. On montre que la diversité des systèmes d'activités et de production, dont on propose une typologie, est principalement liée à la distance à la ville et à l'accès quantitatif et qualitatif à l'eau. On quantifie la fonction alimentaire de cette agriculture, forte pour les produits maraîchers et non négligeable pour le riz. La lutte contre les inondations en plaine et l'érosion dans les collines bâties justifient aussi certaines décisions de maintien local de l'activité agricole, alors que les performances agricoles, quantitatives et qualitatives (risques sanitaires) sont diminuées par les effluents industriels et urbains. La durabilité intrinsèque et exogène de l'agriculture est approchée en première analyse en proposant une hiérarchie entre les systèmes de production et entre les sites. Cependant, les questions liées à la qualité sanitaire variable de certains produits agricoles issus de l'agriculture urbaine, à la segmentation croissante de certaines filières ou aux risques liés à de nouvelles fonctions de l'agriculture ont émergé dans ce projet, mais ouvrent surtout de nouveaux champs de recherche.
\end{abstract}

\begin{abstract}
Urban agriculture in Antananarivo district (Madagascar): an inter-disciplinary approach. An interdisciplinary program (ADURAA) analyzes the sustainability and the urban functions of agriculture inside and around Antananarivo. Its aim is to develop knowledge and to help public decisions about the interest, limits and conditions of the local maintenance of agriculture, compared with other land uses. Six research themes were identified based on a typology of urban farms; they contribute knowledge on three main functions of this agriculture, viz. food supply, land use and risk prevention, and
\end{abstract}

Auteur correspondant: C. Aubry, christine.aubry@agroparistech.fr 
the emerging role of urban waste reuse. A wide diversity of activities systems and agricultural production systems has been identified in relation to distance to the town and water availability. This urban agriculture significantly contributes to the vegetables supply of the district as well as to its rice supply. Flooding in the lowland and erosion in the hills are also efficiently contained by agricultural land use, but anarchic building and the use of agricultural soil for brick making largely counteract this role. Uncontrolled discharge of industrial liquid wastes into the irrigation network greatly reduces rice production and has even led to the desertion of some intra-urban rice-growing areas, while domestic pollution strongly affects the sanitary quality of intra-urban vegetables. However a positive externality could be developed by using some urban solid waste as fertilisers for the growing horticultural sector. Emerging questions such as the quality of urban produce and potential new risks open up new avenues for future research on this agriculture.

L'intérêt des scientifiques pour l'agriculture dite « urbaine » est récent (Bryant et Johnston, 1992 ; Smit et Nasr, 1992 ; Charvet, 1994) et croissant (Nasr et Padilla, 2004; Mougeot, 2005). Il provient des interrogations, corrélatives du mouvement mondial d'urbanisation, sur les rôles de l'agriculture dans le développement durable des villes (Monédiaire, 1999) : on appelle ici agriculture urbaine «l'agriculture localisée dans la ville et sa périphérie, dont les produits sont au moins partiellement destinés à la ville et pour laquelle il existe une alternative entre usage agricole et urbain non-agricole des ressources, [qui] ouvre sur des concurrences mais également des complémentarités possibles entre ces usages » (Moustier et Mbaye, 1999). Le questionnement sur l'intérêt et les conditions de pérennisation in situ d'une occupation agricole de l'espace n'est pas trivial : l'agriculture urbaine est en effet marquée par le partage de ressources productives (foncier, main-d'œuvre, eau, etc.) avec les activités urbaines et par des interactions multiformes avec la ville, positives (proximité du bassin de consommation et d'emploi, accessibilité des services, des intrants) et négatives (conflits fonciers, de voisinage, suspicions sur les produits, etc.). La clarification des fonctions qu'elle remplit permet de mieux expliciter le rôle de l'agriculture urbaine dans la croissance des villes (Donadieu et Fleury, 2003). Ces fonctions sont depuis toujours multiples : alimentaires (Egzabhier et al., 1994; Snrech, 1997), environnementales (prévention/absorption de risques, assainissement de la ville par l'utilisation de ses déchets), paysagères, socio-éducative (Temple et Moustier, 2004). Leur reconnaissance par les politiques publiques est plus récente et variable (Fleury, 2005), notamment dans les pays du Sud (Losch, 2002). L'analyse de ces fonctions et de leur prise en compte par les urbains est donc un enjeu scientifique et opérationnel fort.

À Antananarivo, capitale de Madagascar, ville tropicale de montagne, l'habitat s'est traditionnellement concentré sur les collines et les sommets, en laissant les vallons et les zones de plaine à l'agriculture. Aujourd'hui, celle-ci est présente jusqu'au centre-ville (Photos 1 et 2), où elle continue d'occuper les bas-fonds les plus inondables, la plaine environnante, récemment réaménagée hydrauliquement, et les collines périurbaines : elle représente $43 \%$ des quelque $425 \mathrm{~km}^{2}$ de l'agglomération (Rahamefy et al., 2005). Cependant, elle reste très méconnue et n'est

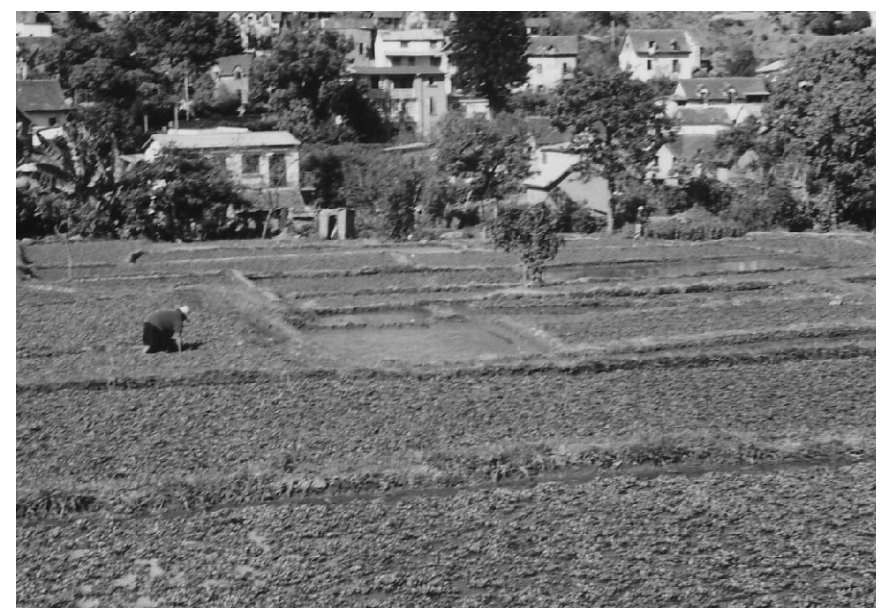

Photo 1. Cressonnières dans le vallon d'Ambanidia, à Antananarivo-ville (centre-ville à 1,5 km). (Photo C. Aubry.)



Photo 2. Plaine rizicole d'Antananarivo le long de la route-digue (centre-ville à $3 \mathrm{~km}$ ). (Photo C. Aubry.)

l'objet d'aucun encadrement technique. Or, l'urbanisation et l'industrialisation rapides de la capitale malgache la concurrencent directement (remblais sur les terres agricoles, habitat formel et informel, rejets polluants dans l'eau à usage agricole) et la rénovation en cours des plans d'urbanisme interroge directement le devenir des espaces agricoles (Cities Alliance, 2004). 


\section{L'agriculture urbaine à Antananarivo : questions de recherche}

À travers un projet de recherche CORUS (Coopération pour la recherche universitaire et scientifique) intitulé ADURAA (Analyse de la durabilité de l'agriculture dans l'agglomération d'Antananarivo) ${ }^{1}$, nous avons posé comme question centrale celle de la durabilité de cette agriculture et des ses modes d'adaptation aux conditions changeantes de son environnement physique et socioéconomique, notamment en termes d'accès aux ressources et de débouchés. Nous appellerons ici durabilité, en cohérence avec la bibliographie existante (CMED, 1987; Jollivet et Pavé, 1993 ; Sachs et Weber, 1994), une agriculture qui soit économiquement viable, socialement vivable et qui préserve les ressources pour le présent et pour le futur.

Dans le contexte urbain, on considérera que la durabilité de l'agriculture renvoie à deux éléments essentiels : (i) les conditions de pérennisation in situ de l'occupation agricole de l'espace, la construction étant un phénomène largement irréversible; (ii) la contribution de l'agriculture au développement durable de la ville, à travers les fonctions qu'elle remplit ou peut remplir pour les urbains. La planification spatiale se réfère désormais à des projets urbains ou territoriaux dans lesquels l'agriculture pourrait prétendre à un rôle au nom de sa multifonctionnalité (Donadieu et Fleury, 2003 ; Fleury, 2005). Nous avons posé comme première hypothèse de travail qu'il existe deux volets contribuant à la durabilité de l'agriculture urbaine (Godard et Hubert, 2002) :

(i) une durabilité "intrinsèque », liée aux conditions mêmes du fonctionnement des exploitations (sontelles viables, vivables? quelles ressources utilisentelles?), dont on peut rendre compte à travers des études de la diversité du fonctionnement technicoéconomique de ces exploitations;

(ii) une durabilité « exogène » qui a trait aux projets que « la ville» (ses planificateurs, ses résidents, etc.) ont sur l'agriculture : intrinsèquement «durable », une agriculture peut néanmoins être condamnée par un

\footnotetext{
1 ADURAA est un projet de recherche-formation (2003-2006) soutenu par le ministère français des Affaires étrangères (programme CORUS); il est né du questionnement conjoint sur le devenir de l'agriculture urbaine par des chercheurs et des enseignants français et malgaches et par les instances territoriales (commune urbaine d'Antananarivo [CUA], communes environnantes FIFTAMA, Région) confrontées à la révision des plans d'urbanisme. Il a pris naissance pendant la crise politique de 2002, qui a mis en exergue l'importance de l'approvisionnement de proximité. Un partenariat étroit, manifesté par des participations croisées aux comités de pilotage et aux réunions de travail, est construit avec les instances territoriales et les ministères concernés. Vingt-trois mémoires de DEA ou d'ingénieur au moins ont été réalisés dans le cadre d'ADURAA et trois thèses ont été soutenues (géographie, agronomie, chimie).
}

projet urbain d'infrastructure; a contrario, « la ville» peut souhaiter maintenir des espaces agricoles où les exploitations sont non viables per se.

C'est donc par le croisement de ces deux «durabilités » que l'on peut se prononcer sur la durabilité globale de l'agriculture urbaine. Nous avons alors décliné des hypothèses portant sur la diversité de cette agriculture et ses conditions de durabilité.

1. Les conditions de durabilité « exogène » de l'agriculture sont liées en premier lieu aux fonctions que les urbains lui reconnaissent ou peuvent lui reconnaître : fonctions d'approvisionnement alimentaire de la ville, de prévention de risques, paysagères, récréatives, etc., qui seules ou, le plus souvent, combinées peuvent conduire les urbains à souhaiter la préserver in situ. Il s'agit donc dans le projet ADURAA d'élaborer des connaissances sur ces fonctions dans le cadre local.

2. La durabilité « intrinsèque » renvoie à des critères de performance technico-économique et à l'analyse de la mobilisation des ressources par l'agriculture : elle est donc par hypothèse variable selon les types de système de production et selon la combinaison locale des ressources dans les exploitations; connaître ces types d'exploitation et leur diversité dans le contexte local est donc une étape nécessaire à l'instruction de la question globale.

3. Plus précisément, en référence au contexte géographique d'Antananarivo, la diversité, les performances et les problèmes des systèmes agricoles, ainsi que les fonctions que l'agriculture peut remplir, sont déterminés par deux facteurs majeurs :

- l'accès des exploitants à la ressource en eau, quantitativement et qualitativement, dans un contexte où la riziculture et le maraîchage, cultures fortement consommatrices d'eau, sont dominants, où le climat tropical d'altitude est marqué par une alternance saison des pluies (novembre-mars) / saison sèche (avriloctobre), et où la ressource en eau agricole est menacée en quantité et en qualité par les besoins et les rejets urbains et industriels ;

- la distance et l'accessibilité de la ville : la proximité de la ville favorise l'écoulement des produits (notamment par la vente directe) et peut ainsi entraîner une dynamisation de l'agriculture de proximité (Cour, 2004); elle exerce une pression foncière sur l'agriculture, une éventuelle compétition pour l'accès à la main-d'œuvre, mais peut aussi fournir des revenus par les possibilités d'emploi en ville pour les ménages agricoles.

Il s'ensuit que les formes d'utilisation de l'espace, et notamment les utilisations agricoles, dépendent par hypothèse de la combinaison locale des ressources, en particulier la ressource en eau, et des facteurs de distance et d'accessibilité de la ville. Notre objectif scientifique est donc d'instruire la question de la durabilité de l'agriculture d'Antananarivo au travers de sa connaissance 


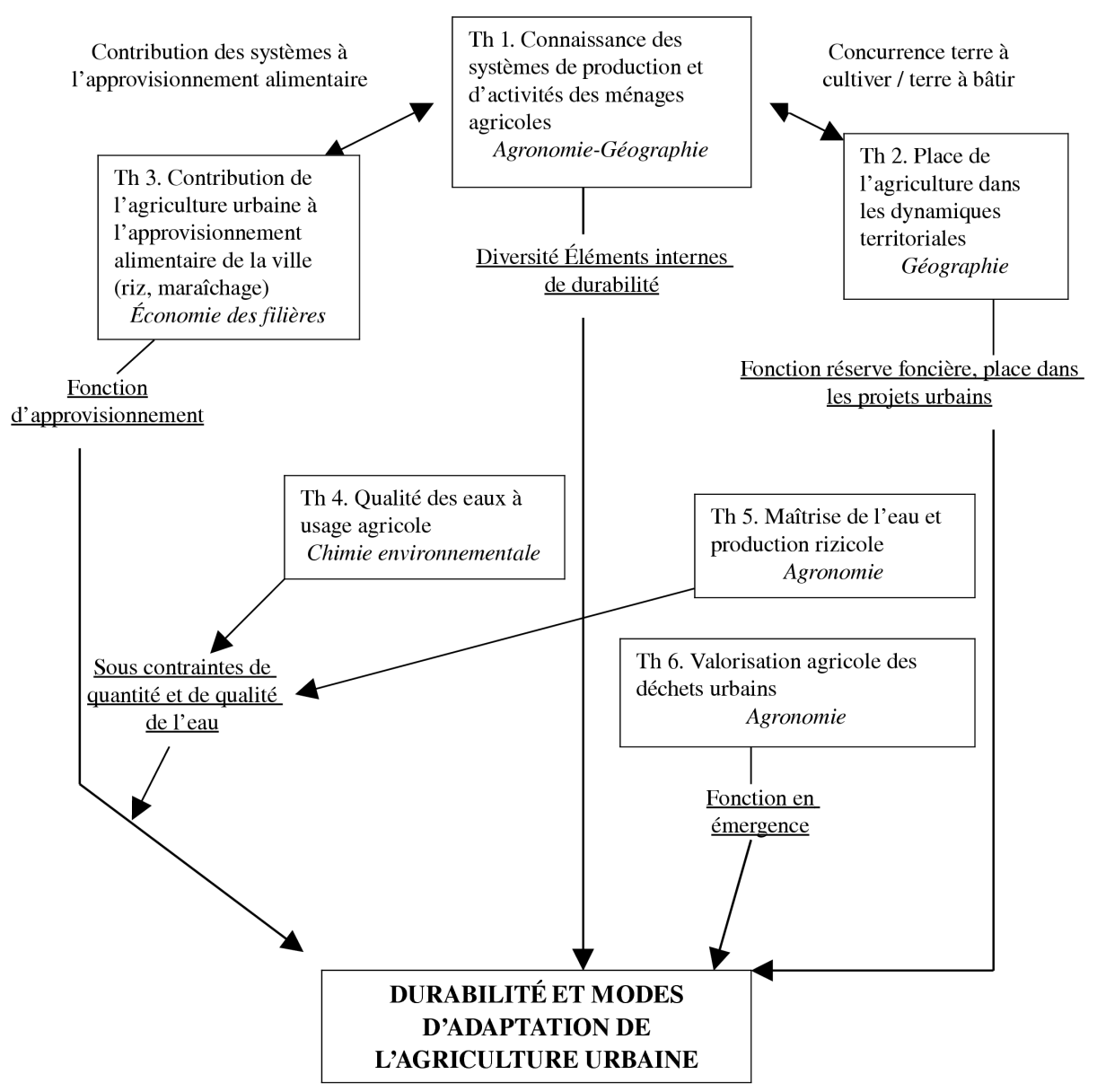

Fig. 1. L'instruction de la question de la durabilité de l'agriculture dans le projet ADURAA.

intrinsèque et du prisme des fonctions qu'elle remplit ou peut remplir pour la ville. L'objectif opérationnel du projet est de contribuer à éclairer les décideurs sur l'intérêt de maintenir l'agriculture par rapport à d'autres formes d'utilisation de l'espace, intérêt a priori variable selon les sites et les types d'agriculture.

\section{Un dispositif de recherches interdisciplinaire et comparatif}

Pour répondre à ces questions de recherche et à ces objectifs, nous avons monté un dispositif pluridisciplinaire : les disciplines sont mobilisées pour apporter des connaissances sur la diversité de l'agriculture, ses facteurs explicatifs et les conditions de durabilité « intrinsèque », ainsi que pour éclairer les différentes fonctions, actuelles ou potentielles, de l'agriculture urbaine.

Les fonctions que nous avons privilégiées, après une première année d'analyse, sont celles d'approvisionnement alimentaire de la ville, de réserve foncière pour l'extension urbaine, et la fonction en émergence de valorisation des déchets urbains. Ces différentes fonctions se déclinent, compte tenu du contexte, sous contrainte majeure de la maîtrise de l'eau, en quantité et en qualité (pollutions industrielles et urbaines). C'est ainsi que nous avons mobilisé l'agronomie pour analyser les aspects techniques de la production (à l'échelle de la parcelle et de l'exploitation), la géographie pour comprendre les rôles de l'agriculture dans les dynamiques spatiales, l'économie des filières pour approcher la fonction d'approvisionnement alimentaire de la ville et la chimie de l'environnement pour quantifier les externalités négatives de la ville sur l'agriculture (pollutions urbaines et industrielles). La figure 1 récapitule la structuration du projet autour de six thèmes, chacun étant porté surtout par une discipline, mais toujours en relation étroite avec une ou plusieurs autres. L'articulation entre ces disciplines a été réalisée selon trois modalités complémentaires :

- une référence commune à une représentation partagée de la diversité de l'agriculture, via une typologie des exploitations élaborée comme base commune ;

- le choix de sites d'étude communs, choisis sur la base de la variation des facteurs majeurs de différenciation retenus : dans ces sites, plusieurs disciplines éclairent 
conjointement une fonction ou une question qui s'y trouvent particulièrement posées. Ainsi, chimie de l'environnement et agronomie ont quantifié dans de mêmes lieux les pollutions industrielles et urbaines des eaux et leurs répercussions sur la production quantitative et qualitative du riz; dans les bas-fonds intra-muros, agronomie et géographie ont analysé ensemble les modes d'occupation agricole de l'espace et leurs adaptations aux forts risques d'érosion et d'inondation. Neuf sites dans l'agglomération (Fig. 2) ont ainsi fait l'objet d'enquêtes pour établir la typologie commune et d'études pluridisciplinaires ;

- un objet $\mathrm{d}$ 'interface où les disciplines apportent une complémentarité méthodologique. Cet objet peut être physique (le sol et son niveau de pollution chimique, interface entre les eaux polluées par des rejets industriels et les plants de riz) ou constituer un système de production agricole. Ainsi, les systèmes rizicoles de plaine sont étudiés par l'économie dans leur rôle d'approvisionnement alimentaire, par la géographie $\mathrm{du}$ fait de leur perturbation par les remblais urbains, par l'agronomie dans leur fonction productive et son altération par les activités para-agricoles (briqueterie, par exemple); les systèmes cressonniers intra-muros sont porteurs d'interrogations spécifiques sur leur durabilité en raison de leur caractère rémunérateur, d'une part, de risques sanitaires potentiels (pollution urbaine) et de menaces par les projets urbains d'infrastructures, d'autre part.

Dans chaque discipline, les méthodes mises en œuvre ont dû faire face au même problème de rareté de données initiales (Encadré). En conséquence, l'étude ici réalisée ne prétend pas à un caractère d'exhaustivité, mais plus à une mise en évidence de l'existence d'une diversité spatialement située et de problèmes de durabilité variés : le projet ADURAA s'est d'emblée présenté comme défrichant une problématique localement nouvelle et devant déboucher sur des études d'approfondissement.

Après avoir présenté la diversité des formes d'agriculture à Antananarivo, nous montrerons certains résultats illustrant des fonctions particulières ou mettant en évidence le fonctionnement pluridisciplinaire du projet. Nous conclurons par une discussion sur les conditions de durabilité de cette agriculture telles que nous les saisissons à l'issue du projet.

\section{La diversité de l'agriculture dans l'agglomération : places dans les systèmes d'activités des ménages}

Une phase initiale a consisté à organiser la diversité constatée des exploitations agricoles et à la relier aux facteurs de différenciation retenus a priori (accessibilité de la ville, disponibilité de l'eau). Nous avons donc élaboré une typologie basée sur des enquêtes de fonctionnement d'exploitations, dans l'ensemble des sites choisis.

La proximité urbaine favorisant la diversité des activités des ménages (Mougeot, 1995 ; Bryant, 1997), nous avons dépassé la vision classique de l'agronome axée sur les seuls systèmes de production agricole pour intégrer, à travers la notion de systèmes d'activités (Laurent $e t$ al., 1994), une vision plus économique et géographique. Nous analysons la place qu'occupe l'agriculture dans les activités (temps, revenus) et les stratégies de ces ménages «agri-urbains ». La typologie construite est alors un croisement entre des types de systèmes d'activités et des types de systèmes de production agricole. L'analyse de ces enquêtes dans les différents sites aboutit (Tab.1) à un double constat (Ramamonjisoa et al., 2007) :

1. L'existence de trois groupes dominants de systèmes d'activités $(A, B, C)$ : en $A$, les ménages se consacrent aux activités agricoles et para-agricoles ${ }^{2}$; en $B$, le chef d'exploitation est à temps plein sur ces activités et au moins un membre du ménage a une activité extérieure ; en $C$, le chef d'exploitation a une activité extérieure au moins à mitemps. Les activités para-agricoles ou extérieures sont très liées à la demande de la ville et aux opportunités qu'elle offre : vente directe de produits agricoles sur des marchés plus ou moins formels (maraîchage, petit élevage); salariat dans les industries, les services, artisanat lié à la construction (menuisiers, charpentiers) ou vendu en ville (broderies) ; fabrication de briques pour la construction urbaine dans les parcelles rizicoles argileuses. Ces trois groupes sont présents dans tous les sites mais inégalement répartis : le $C$ est plus fréquent lorsque la ville est plus facilement accessible (route, bus), comme on peut le noter dans les deux sites collinaires du tableau 1. L'une de nos hypothèses sur les facteurs de différenciation est ainsi vérifiée. Une constante est le faible réinvestissement de ces revenus extérieurs dans l'agriculture : ils servent à la survie de la famille, rarement à l'achat d'intrants ou à la rémunération de salariés agricoles et exceptionnellement à l'achat de foncier.

2. Au sein de ces systèmes d'activités, les systèmes de production agricole sont diversifiés et déterminés d'abord, en accord avec l'une des hypothèses, par l'accès à l'eau : riz dominant en zones inondables, maraîchage ailleurs lorsque l'eau est accessible, fréquente présence de petits élevages (canards, oies, volailles, porcs) ou de quelques vaches laitières. Ces systèmes de production sont très liés aux systèmes d'activités : dans les sites collinaires à dominante maraîchère, les «doubles-actifs » (les « $C$ ») produisent surtout des cultures maraîchères à cycle court, demandant peu de travail et d'intrants (haricots verts,

\footnotetext{
${ }^{2}$ On appelle ici «para-agricole » une activité qui utilise les moyens de production de l'exploitation (attelage de bœufs, salariat agricole, etc.) ou les productions elles-mêmes (vente directe) pour en tirer un revenu supplémentaire.
} 
Plaine rizicole : 3 sites

Intra muros : 3 vallons

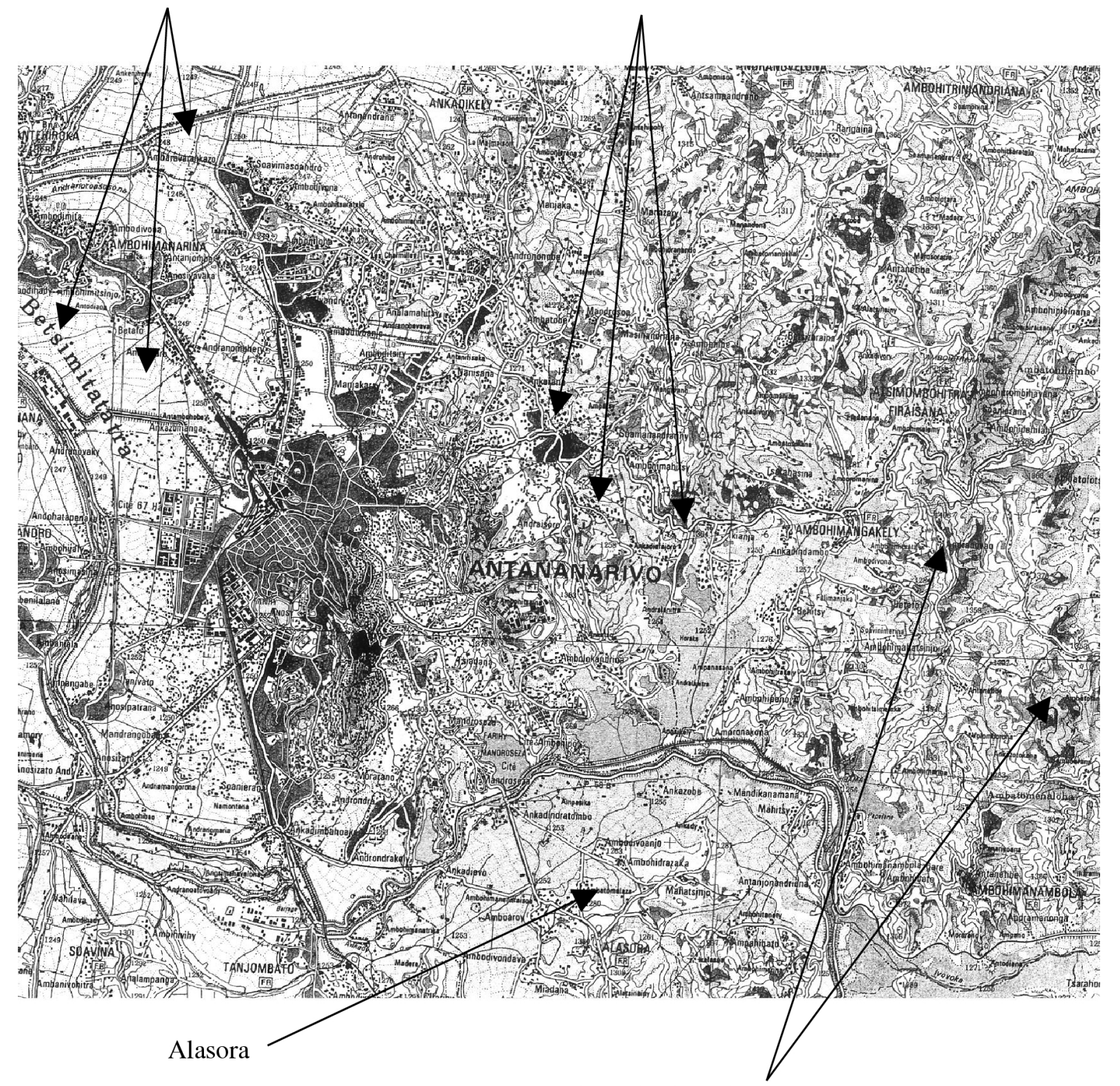

Collines de l'Est : 2 sites

Fig. 2. Choix des sites d'études dans l'agglomération d'Antananarivo pour le projet ADURAA.

légumes-feuilles très prisés localement, appelés brèdes); les types $\mathrm{A}$ ont des systèmes de culture maraîchère plus diversifiés (alternance de cycles courts et de cycles longs, comme tomate, chou-fleur, etc.), conduits de façon plus intensive et souvent associés à un petit élevage laitier, producteur de lait vendu en ville mais aussi de fumier pour le maraîchage à cycle long. Un système souvent rencontré en plaine est, sur une surface de moins de 0,5 hectare, la combinaison «riz, canards, pêche, briques », qui illustre la multifonctionnalité du sol agricole lui-même : il fait se succéder sur chaque parcelle, au cours d'une année, le riz de septembre à février, l'élevage de canards et la pêche pendant la longue période de drainage (février-juin) et la fabrication de briques après drainage (juillet à septembre) à partir de l'horizon de surface argileux des rizières. Cette dernière activité est lucrative (20 000 à 25000 briques sur un are de rizière peuvent rapporter mensuellement 3 à 4 fois le salaire d'un ouvrier industriel), mais mine le sol : une rizière «à double fonction » ne dure ainsi que 3 à 5 ans, avant d'atteindre les couches sous-jacentes du sol (gley, pseudogley), impropres tant à la briqueterie qu'à la riziculture. Le système « riz, canards, pêche, briques » est ainsi, par essence, non durable. Cette diversité n'exclut pas quelques points communs : le riz, par exemple, est 


\section{Encadré 1. Méthodologies face à l'absence de données initiales}

Pour les enquêtes globales par site ${ }^{a}$, en l'absence de tout recensement agricole, nous avons eu recours, pour échantillonner, à des personnes-ressources (maires, présidents de fokontany ${ }^{b}$, notables, etc.) nous indiquant des agriculteurs sur la base de critères que nous précisions, et à la délimitation géographique de petites zones (hameaux, vallon) où l'exhaustivité était alors recherchée. Dans les zones plus importantes, la longueur des enquêtes et les moyens disponibles ont conduit à retenir environ 20 ménages par site. Au total, nous avons réalisé dans le projet un peu plus de 200 enquêtes. De même, la rareté des statistiques disponibles pour les filières d'approvisionnement de la capitale a conduit à (i) mobiliser de façon pragmatique la «boîte à outils » du praticien de l'analyse de filières (nombreuses enquêtes par échantillons raisonnés, graphe des flux, tableau d'analyse fonctionnelle, matrice des marchés, etc.), (ii) recueillir des informations primaires, amenant à cibler certains produits seulement, (iii) prendre en compte la « consommation » (300 à 400 enquêtes de consommateurs par étude, choisis pour leur diversité socioéconomique) et la dimension spatiale, pour mieux comprendre la diversité de la fonction d'approvisionnement. En géographie, nous avons recouru aux données cadastrales disponibles et à de nombreux relevés de terrain (découpages parcellaires, statuts fonciers, occupations du sol), ce qui a nécessairement limité l'extension géographique des sites analysés. Pour estimer les risques d'inondation et d'érosion, nous avons recueilli des indicateurs variés mais simples d'appréhension, dont nous avons recherché la convergence : analyse diachronique de photos aériennes, dont des relevés précis de courbes de niveau et de progression des lavakac et des ensablements; dispositifs simples de mesure in situ des quantités de terre accumulées en fond de vallée après les fortes pluies estivales; enquêtes auprès de la commune (fréquences d'éboulements, coupures de routes, etc.). En agronomie, à l'échelle de la parcelle, nous avons recueilli des données de rendement lors des enquêtes globales d'exploitation, mais aussi procédé directement à un diagnostic agronomique de l'élaboration du rendement du riz sur des parcelles choisies pour présenter un gradient de maîtrise quantitative ou qualitative de l'eau (dans ce cas en concertation avec la chimie) ${ }^{d}$. En chimie, l'impossibilité de connaître précisément les polluants industriels a conduit à privilégier des indicateurs globaux (aspect, turbidité, acidité, conductivité électrique, demande chimique ou biologique en oxygène) et à les comparer à des normes internationales sur la qualité des eaux d'irrigation. Les prélèvements ont été effectués aux émissaires des usines, dans les canaux d'irrigation, dans les rizières choisies en commun avec les agronomes, à des moments pour partie communs (mise en eau des rizières, tallage) et pour partie liés à des objectifs propres (variabilité journalière ou saisonnière des rejets).

${ }^{a}$ L'enquête porte sur la composition de la famille, les activités des membres (nature, temps et position dans l'année, part dans le revenu et relations avec les activités agricoles), l'histoire de l'exploitation, le milieu, le système de production agricole (taille, statuts fonciers, combinaison des productions, modes de conduite technique, destination des produits) et sur les perspectives de pérennité du système d'activités et du système de production agricole.

${ }^{b}$ Découpage administratif proche du quartier.

Figures d'érosion marquant le paysage.

${ }^{d}$ Sur quatre rizières par site, choisies selon un gradient de proximité vis-à-vis des sources polluantes, les agronomes analysent l'élaboration du rendement du riz, son développement et des états du milieu (sol, climat), mais complètent par des analyses spécifiques du sol (dont la conductivité et la teneur en métaux lourds) et de plantes (polluants potentiels).

très largement autoconsommé, seuls quelques types d'exploitation en commercialisant régulièrement. La culture du cresson dans les bas-fonds intra-muros est associée ou non à d'autres activités : elle est souvent conduite de façon intensive (nombreux cycles successifs, utilisation de nombreux intrants) et très rémunératrice pour les agriculteurs, car il existe une forte demande de cresson dans la ville et la production est majoritairement vendue en circuit court.

\section{Une fonction alimentaire importante passant par des filières diversifiées}

L'analyse de cette fonction a porté sur (i) l'ajustement entre la demande et l'offre sur les marchés d'Antananarivo (quantité, qualité, diversité, disponibilité, préférences et critères de choix) et (ii) l'analyse des filières de certains produits phare de l'agriculture urbaine (structure, fonctionnement, dynamique). Nous avons fait l'hypothèse que la localisation spatiale des productions et la longueur des circuits de commercialisation influent sur les performances des filières. Les produits retenus (riz et maraîchage, dont la tomate et, plus récemment, le cresson) sont significatifs dans l'alimentation locale et illustrent une diversité de rôles dans l'approvisionnement et les circuits de distribution.

Une part significative de l'approvisionnement en riz de l'agglomération (environ 174000 tonnes/an) provient de l'agriculture urbaine : elle représente 24000 tonnes (14\%) essentiellement autoconsommées et jusqu'à 15000 tonnes mises sur le marché certaines années par certains types d'exploitation. L'autoconsommation touche partout la famille paysanne élargie, y compris des membres vivant en ville (Dabat et al., 2004); par contre, ce sont surtout les grosses exploitations de la plaine (types CP1 et AP1 : cf. Tab. 1) qui contribuent le plus à la vente de riz sur le marché. En maraîchage, le chiffre d'affaires des filières approvisionnant Antananarivo avoisine 100000 euros par an, celui du riz étant de 220000 euros : outre ce poids économique, en forte croissance, le maraîchage local a une part importante dans l'approvisionnement de la ville (exclusive pour des produits périssables comme les brèdes 
Tableau 1. Systèmes d'activités et systèmes de production agricole dans quelques sites de l'agglomération d'Antananarivo.

\begin{tabular}{|c|c|c|c|c|}
\hline $\begin{array}{c}\text { Sites intra-muros } \\
\text { (nombre } \\
\text { d'exploitations) }\end{array}$ & Surface et statut & $\begin{array}{l}\text { Productions } \\
\text { végétales }\end{array}$ & $\begin{array}{l}\text { Productions } \\
\text { animales }\end{array}$ & $\begin{array}{l}\text { Main-d'œuvre - } \\
\text { Équipement }\end{array}$ \\
\hline AI1 (3) & $\begin{array}{l}2 \text { à } 3 \text { ha } \\
\text { Location et propriété }\end{array}$ & $\begin{array}{l}\text { Cresson, maraîchage, } \\
\text { riz }\end{array}$ & Volailles, porcs & $\begin{array}{l}\text { Familiale, salarié } \\
\text { temporaire - } \\
\text { Manuel et } \\
\text { pulvérisateur à dos } \\
\text { en propriété }\end{array}$ \\
\hline BI1 (15) & $\begin{array}{l}1 \text { à } 2 \text { ha } \\
\text { Location, métayage }\end{array}$ & $\begin{array}{l}\text { Cresson, } \\
\text { (maraîchage) }\end{array}$ & (volailles) & $\begin{array}{l}\text { Familiale seule - } \\
\text { Manuel }\end{array}$ \\
\hline CI1 (2) & $\begin{array}{l}1 \text { à } 4 \text { ha } \\
\text { Propriété }\end{array}$ & $\begin{array}{l}\text { Riz, maraîchage, } \\
\text { (cresson) }\end{array}$ & 2 à 3 vaches laitières & $\begin{array}{l}\text { Salariés permanents - } \\
\text { Attelages, } \\
\text { pulvérisateurs à dos }\end{array}$ \\
\hline CI2 (10) & $\begin{array}{l}2 \text { à } 5 \text { a } \\
\text { Métayage seul }\end{array}$ & Cresson seul & - & $\begin{array}{l}\text { Familiale seule - } \\
\text { Manuel seul }\end{array}$ \\
\hline \multicolumn{5}{|l|}{ Plaine rizicole (idem) } \\
\hline AP1 (3) & $\begin{array}{l}30 \text { à } 70 \text { a } \\
\text { Propriété (Location) }\end{array}$ & Riz & $\begin{array}{l}\text { Bœufs (2 à 7) } \\
\text { Volailles (20 à 40) }\end{array}$ & $\begin{array}{l}\text { Familiale + salarié } \\
\text { temporaire - } \\
\text { Attelage, outils }\end{array}$ \\
\hline AP2 (11) & $\begin{array}{l}3 \text { à } 10 \text { a } \\
\text { Propriété }\end{array}$ & $\begin{array}{l}\text { Riz, manioc, pomme } \\
\text { de terre } \\
\text { (contre-saison) }\end{array}$ & $\begin{array}{l}\text { Bœufs (2 à 8), } \\
\text { volailles (20 à 65), } \\
\text { porcs (> 5) }\end{array}$ & $\begin{array}{l}\text { Familiale - } \\
\text { Manuel }\end{array}$ \\
\hline AP3 (5) & 10 à $20 \mathrm{a}$ & Riz & Volailles $(<15)$ & $\begin{array}{l}\text { Familiale - } \\
\text { Manuel }\end{array}$ \\
\hline BP1 (9) & 10 à $20 \mathrm{a}$ & Riz & - & Familiale \\
\hline CP1 (2) & $\begin{array}{l}70 \text { a à } 1 \text { ha } \\
\text { Propriété }\end{array}$ & Riz & $\begin{array}{l}\text { Bœufs (5 à 7), } \\
\text { volailles (25 à 75) }\end{array}$ & $\begin{array}{l}\text { Familiale + salarié } \\
\text { permanent - } \\
\text { Attelage, outils }\end{array}$ \\
\hline CP2 (10) & $\begin{array}{l}2 \text { à } 15 \text { a } \\
\text { Métayage }\end{array}$ & Riz & Volailles $(<25)$ & $\begin{array}{l}\text { Familiale + salarié } \\
\text { temporaire - } \\
\text { Manuel }\end{array}$ \\
\hline
\end{tabular}

A, B, C : groupes de systèmes d'activités ; I = intra-muros, $\mathrm{P}=$ plaine.

ou le chou-fleur), avec certaines spécialisations de sites : leur complémentarité de production dans le temps permet un étalement de la commercialisation des légumes et un lissage des prix de certains produits. C'est le cas pour la tomate (N’Dienor et al., 2006), qui passe de la catégorie des produits saisonniers à celle des produits de toute saison, du fait de la diversité des zones d'approvisionnement et de leur plurisaisonnalité (de 2 à 4 saisons de production selon les sites). Cette étude a permis de quantifier la consommation et de préciser la place de l'agriculture urbaine dans cet approvisionnement ${ }^{3}$ (Tab. 2).

Une analyse comparative sur les différentiels de coûts de production liés à la proximité de la ville a été menée sur riz et tomate : elle montre que la localisation périurbaine est la plus intéressante pour ces deux cultures, l'intraurbain étant, pour le riz, très handicapé par les problèmes fonciers, le coût de la main-d'œuvre et l'irrégularité de

\footnotetext{
${ }^{3} 44 \mathrm{~kg}$ de tomate consommés par habitant de la capitale et par an de façon convergente dans notre étude, alors que les statistiques disponibles, anciennes, indiquent environ $20 \mathrm{~kg} / \mathrm{hab} / \mathrm{an}$.
}

la maîtrise de l'eau et le rural éloigné l'étant par le coût du transport. Pour la tomate, le rural plus éloigné souffre en outre des risques importants de perte de produit lors du transport, l'intra-urbain est très rare. On montre ainsi l'efficacité économique de certaines formes d'agriculture urbaine, en localisant des zones optimales de production du point de vue du coût des facteurs de production.

Cette localisation préférentielle est bien comprise par les agriculteurs : agronomes et géographes du projet ont montré que, face à l'augmentation de la demande des urbains en légumes et de la pression foncière sur les bas-fonds, les agriculteurs des collines périurbaines colonisent en maraîchage les terres ferrallitiques de pente (tanety), autrefois consacrées aux cultures vivrières en sec (manioc, patate douce) ou au parcours des bovins de trait. Cette dynamique spatiale récente (une dizaine d'années) s'accompagne, pour les agriculteurs, d'une construction pluriannuelle de la fertilité de ces tanety pauvres, qui mobilise beaucoup de fumier et d'engrais. 
Tableau 2. Résultats partiels de rendements en tomate (t/ha) dans l'expérimentation utilisant des déchets urbains (N’Dienor, 2006). Première campagne : octobre 2003-février 2004.

\begin{tabular}{|c|c|c|c|}
\hline \multirow{2}{*}{$\begin{array}{l}\text { Numéro et nom local des sols } \\
\text { 1. Tanety-Baïboho }\end{array}$} & \multicolumn{3}{|c|}{$\begin{array}{l}\text { Rendement tomate (t/ha) et écart-type ( ) des } 6 \text { poquets de tomate } \\
\text { suivis par traitement }\end{array}$} \\
\hline & $\begin{array}{c}\text { T1 (fumier au trou }+ \\
\text { engrais en } \\
\text { couverture) }\end{array}$ & $\begin{array}{c}\text { T3 (terreau au trou }+ \\
\text { engrais en } \\
\text { couverture) }\end{array}$ & $\begin{array}{c}\text { T4 (fumier au trou }+ \\
\text { terreau en } \\
\text { couverture) }\end{array}$ \\
\hline 2. Tanety-Baïboho & $\begin{array}{c}28 \\
(7,30) b\end{array}$ & $\begin{array}{c}38 \\
(7,84) \mathrm{a}\end{array}$ & $\begin{array}{c}27 \\
(7,62) b\end{array}$ \\
\hline 3. Tanety-Baïboho & $\begin{array}{c}41 \\
(12,40) a\end{array}$ & $\begin{array}{c}43 \\
(13,2) a\end{array}$ & $\begin{array}{c}25 \\
(10,5) b\end{array}$ \\
\hline 4. Baïboho & $\begin{array}{c}37 \\
(10,9) \mathrm{a}\end{array}$ & $\begin{array}{c}35 \\
(9,37) \mathrm{a}\end{array}$ & $\begin{array}{c}37 \\
(11,7) \mathrm{a}\end{array}$ \\
\hline 5. Tanety rouge & $\begin{array}{c}39 \\
(11,98) b\end{array}$ & $\begin{array}{c}50 \\
(8,33) a\end{array}$ & $\begin{array}{c}38 \\
(15,08) b\end{array}$ \\
\hline 6. Tanety ocre & $\begin{array}{c}13 \\
(7,37) a\end{array}$ & $\begin{array}{c}16 \\
(2,88) a\end{array}$ & $\begin{array}{c}19 \\
(4,83) a\end{array}$ \\
\hline 7. Baïboho & $\begin{array}{c}22 \\
(11,19) \mathrm{a} \\
\end{array}$ & $\begin{array}{c}26 \\
(5,74) \mathrm{a} \\
\end{array}$ & $\begin{array}{c}31 \\
(6,67) \mathrm{a} \\
\end{array}$ \\
\hline 8. Tanety-Baiboho & $\begin{array}{c}72 \\
(16,34) \mathrm{ab}\end{array}$ & $\begin{array}{c}91 \\
(13,65) \text { a }\end{array}$ & $\begin{array}{c}79 \\
(22,08) \mathrm{ab}\end{array}$ \\
\hline 9. Tanety «âgée », adaptée au maraîchage & $\begin{array}{c}44 \\
(14,21) \mathrm{a}\end{array}$ & $\begin{array}{c}40 \\
(4,21) \mathrm{a}\end{array}$ & $\begin{array}{c}30 \\
(9,56) \mathrm{b}\end{array}$ \\
\hline 10. Baiboho & $\begin{array}{c}54 \\
(12,32) \mathrm{a}\end{array}$ & $\begin{array}{c}53 \\
(20,38) a\end{array}$ & $\begin{array}{c}46 \\
(19,92) \mathrm{ab}\end{array}$ \\
\hline 11. Tanety-Baïboho & $\begin{array}{c}53 \\
(10,33) a b\end{array}$ & $\begin{array}{c}63 \\
(13,47) a\end{array}$ & $\begin{array}{c}60 \\
(8,66) a\end{array}$ \\
\hline
\end{tabular}

En gras et en italique : sol-type de sa catégorie; a, b, c : groupes homogènes selon le test de Newman Keuls à 5 \%

Enfin, la culture du cresson soulève des questions relatives à sa fonction alimentaire : c'est sur ce produit, très prisé des Malgaches, qu'a commencé à être testée l'hypothèse selon laquelle l'attention des consommateurs sur la qualité des produits pourrait être un élément de compétitivité important de l'agriculture urbaine ; en effet, la production intra-muros de cresson à partir d'eaux usées rencontre de plus en plus la méfiance des consommateurs. Le projet ADURAA a montré la segmentation émergente de la filière cresson, initiée tant par des consommateurs individuels privilégiant du cresson produit en périurbain avec de l'eau non polluée que par les grandes et moyennes surfaces en développement rapide à Antananarivo.

\section{Terre à bâtir versus terre à cultiver : une équation... à risques}

La fonction de réserve foncière pour l'urbanisation que joue de fait l'agriculture urbaine est en cours de modification à l'échelle de l'agglomération. L'urbanisation et l'industrialisation se sont jusqu'ici surtout développées dans la plaine facilement aménageable. Les risques récurrents d'inondation, accrus par les remblais anarchiques qui ont fortement perturbé le fonctionnement hydraulique de la plaine, conduisent aujourd'hui l'agglomération à préserver dans les plans d'urbanisme des zones tampons à usage agricole dans cette plaine. Leur utilisation en riziculture irriguée est techniquement peu performante (moins de 2,5 tonnes de paddy à l'hectare en moyenne) en raison d'une faible utilisation d'intrants, de variétés souvent obsolètes, de techniques de production peu efficientes (comme l'utilisation de plants à repiquer âgés, donc à faible capacité de tallage) et d'une gestion collective de l'eau inexistante ou très limitée. Cependant, malgré ces obstacles techniques, la riziculture de plaine garantit, outre une certaine fonction alimentaire (voir ci-dessus), des usages supposés contrôlés du sol et des espaces susceptibles d'absorber l'eau lors des crues et des épisodes de pluies cycloniques. Dans les nouveaux schémas d'urbanisme, on préconise ainsi un maintien de plus de 2000 hectares rizicoles en plaine et le « retour » sur les collines pour l'habitat (et partiellement l'industrie). $\mathrm{Ce}$ « retour » nous interroge à double titre :

1. Le maintien en riziculture de la plaine n'est pas acquis : la briqueterie, qui s'y est fortement développée ces dernières années, conduit à rendre improductives des zones que l'on souhaite aujourd'hui conserver rizicoles. La double utilisation du sol (riz-briques) pendant quelques années semble bien être, pour les propriétaires, une 
stratégie d'anticipation d'une sortie de l'usage agricole. On a ainsi vu, dans une commune périphérique, de nombreux permis de briqueterie, sollicités sous "prétexte» de niveler la rizière pour faciliter la maîtrise de l'eau, se transformer trois ou quatre ans plus tard en remblais de fait, perturbant par ce mitage le projet communal de maintien de la plaine rizicole. Dans les zones de plaine où la riziculture a déjà été abandonnée, nos études en géographie montrent que les marécages à jacinthe d'eau se multiplient, source de nouvelles activités agricoles (la jacinthe est très prisée comme fourrage pour les bovins), mais aussi de possibles risques sanitaires et épidémiologiques qu'il faudrait pouvoir étudier.

2. Construire sur les pentes et dans les bas-fonds est largement pratiqué au sein même de la capitale, mais induit de forts risques d'inondation et d'érosion liés à la situation de ville tropicale d'altitude. Les relations entre habitat d'amont et agriculture d'aval et les risques engendrés ont été étudiés intra-muros comme anticipation possible de ce qui pourrait survenir ailleurs dans l'agglomération (Andriamalala, 2006) : étude détaillée de l'habitat, de l'occupation agricole de l'espace et estimation du rôle d'absorption d'inondation et d'érosion de l'agriculture. On constate une forte convergence entre les indicateurs utilisés, amenant à considérer ces risques d'érosion comme très importants, notamment le phénomène de "lavakisation ", dangereux pour les habitants : ainsi, dans un des vallons, on a montré que les pluies érosives $(>50 \mathrm{~mm} / \mathrm{h}$ ) arrachent environ 3 tonnes de terre par an, et qu'en 5 ans, un lavaka proche des habitations avait crû de plus de $75 \%$ (ibid.). Rizières et cressonnières de ces bas-fonds peuvent par ailleurs stocker une grande quantité d'eau pendant quelques heures à l'aide des diguettes construites par les agriculteurs : un vallon de 287 hectares emmagasine $850000 \mathrm{~m}^{3}$ d'eau, soit 3 jours successifs de très fortes pluies. Ces faits militent pour limiter dans ces bas-fonds les projets en cours de construction d'infrastructures, notamment routières.

La prolifération de l'habitat sur les pentes, sans accompagnement d'infrastructures d'écoulement de l'eau, se répercute aussi directement sur les systèmes agricoles. D'une part, les produits d'érosion arrivant sur les parcelles de bas-fonds les ensablent, amenant les agriculteurs à accroître leur pratique de la briqueterie en bas-fonds, en «comblant les trous » par ces produits d'érosion, changeant ainsi la nature même des sols. D'autre part, les agronomes montrent que l'excès de matières organiques dans les eaux d'assainissement arrivant dans ces basfonds conduit à abandonner la riziculture au profit de la production de cresson ${ }^{4}$. Celui-ci est ainsi pour partie

\footnotetext{
4 Au-delà d'une certaine charge organique des eaux, le riz ne parvient plus à fructification et reste à l'état de prolifération végétative. Le cresson s'accommode fort bien de cette charge organique (niveaux de production doubles de ceux rencontrés en périurbain, où l'eau est « propre »).
}

le produit de l'urbanisation d'amont, et les chimistes montrent qu'il contribue aussi à l'assainissement des eaux d'aval, au détriment de sa propre qualité sanitaire (forte contamination fécale du cresson lorsqu'il pousse dans des parcelles d'eaux usées dominantes). La conservation agricole de ces bas-fonds, qui présente pour la ville un intérêt par l'absorption des inondations et des produits d'érosion, et, pour les agriculteurs, un intérêt économique net, pose des problèmes de santé publique qui interrogent de façon complexe sur leur durabilité « exogène ».

\section{Les contraintes de production liées à la pollution industrielle}

La pollution industrielle des eaux et ses répercussions sur la productivité rizicole, voire l'utilisation même du territoire, a été traitée dans deux sites proches de zones industrielles, en combinant chimie de l'environnement, agronomie à l'échelle de la parcelle et de l'exploitation et géographie(localisation des pollutions et répercussions sur les activités humaines).

Les analyses des rejets montrent des pollutions variables mais pouvant être très fortes et entraîner des dépôts visibles dans les sols (conductivité électrique de l'eau des rizières dépassant $1200 \mathrm{~S} / \mathrm{cm}$, la norme pour les eaux d'irrigation étant inférieure à $250 \mathrm{~S} / \mathrm{cm}$ ). Les résultats agronomiques montrent, en situation de forte pollution, des rendements très diminués (ne dépassant pas 0,13 tonne à l'hectare contre 1,5 à 2 tonnes hors zones polluées) et un cycle végétatif très allongé, conduisant à des attaques parasitaires plus importantes. On note une grande variation interannuelle des répercussions de la pollution sur ces rizières, pour partie classique (climat, conduite technique), mais aussi liée à celle des niveaux de pollution. Début de traitement des eaux par certaines usines et détournement des eaux les plus polluées vers d'autres sites sont deux phénomènes observés... probablement liés à notre présence persistante et visible sur la zone!

Ces situations très contraignantes sont cependant «supportées » par les agriculteurs : les enquêtes d'exploitations et les études menées lors d'un stage en économie de l'environnement montrent que les bénéfices tirés de la double activité paysanne dans les usines et de la vente informelle des produits agricoles locaux aux salariés de celles-ci compensent largement les coûts économiques directs de la pollution industrielle (baisses de rendement, réorientations obligées de la production, etc.). Les effets néfastes des émanations sur la santé sont ainsi souvent évoqués lors des enquêtes, mais sont rarement l'objet de dépôts de plainte. 


\section{La valorisation des déchets urbains : une fonction "en émergence " de l'agriculture urbaine}

Le constat de l'extension spatiale des systèmes maraîchers sur les collines, accompagnée d'une construction longue et coûteuse de la fertilité de ces terres pauvres, nous a amenés à tester l'introduction possible dans les pratiques agricoles d'un criblé de décharge naturellement composté, localement appelé «terreau», auquel certains paysans ont commencé à recourir comme substitut aux matières fertilisantes (fumier et engrais chimiques).

Une étude combinant analyse approfondie des pratiques paysannes et tests au champ de la substitution «terreau » à la place du fumier (traitement T3) et «terreau » à la place des engrais ${ }^{5}$ (traitement T4) a montré, pendant trois campagnes successives sur tomate (Tab. 2), que les traitements utilisant le terreau se comportent aussi bien et souvent mieux (plus forts rendements, plus faibles variabilités intraparcellaires) que les traitements «paysans » T1 (N’Dienor, 2006). Leur effet positif est plus marqué sur les tanety récemment mises en culture dans le cadre de l'extension spatiale du maraîchage. Ce résultat est encourageant pour le terreau, alors qu'il s'agit d'un produit de base, peu cher à produire et en quantité non limitée.

On rejoint de fait une préoccupation des urbanistes et des gestionnaires de l'agglomération quant au devenir de la décharge municipale, aujourd'hui saturée : l'innocuité du terreau peut permettre de transformer, pour partie au moins, ces déchets en ressources pour l'agriculture de proximité. Une communication permanente sur ces résultats avec les décideurs locaux a permis d'accélérer cette prise de conscience : des mesures ont été prises en 2005 pour augmenter la production de terreau par la population vivant sur la décharge et une nouvelle filière est en émergence (Pierrat, 2006).

\section{Discussion : durabilité et fonctions de l'agriculture urbaine}

Le projet ADURAA a apporté de premières réponses à la question générale de la durabilité de cette agriculture urbaine. Nous avons notamment montré son caractère multifonctionnel. Pour les ménages agricoles, l'agriculture est toujours source d'autoconsommation alimentaire, de

\footnotetext{
${ }^{5}$ Le criblé de décharge localement appelé terreau présente une richesse moyenne en matière organique, faible en azote et élevée en phosphore et ses teneurs en métaux lourds sont très inférieures aux nouvelles normes européennes (matières organiques : $14,3 \% ; \mathrm{N}: 0,36 \% ; \mathrm{P}: 0,83 \% ; \mathrm{K}: 1 \%$; ETM (ppm) : $\mathrm{Cd}: 0,72 ; \mathrm{Cu}: 38,3 ; \mathrm{Pb}: 113 ; \mathrm{Zn}: 287 ; \mathrm{Cr}: 53,3 ; \mathrm{Hg}: 2,67$; As : 11,4). Des analyses microbiologiques ont montré en outre l'absence d'agents phytopathogènes.
}

revenu principal (A) ou complémentaire (B, C). L'exploitation constitue toujours un lieu de résidence (cherté des logements en ville) et souvent le lieu de la conservation du patrimoine familial (rizières ancestrales, éventuellement destinées à devenir terrains à bâtir pour la famille après passage éventuel par la briqueterie). Cette agriculture a aussi été un "amortisseur de crise " pendant les événements politiques et économiques de 2002, qui ont isolé l'agglomération pendant près de 6 mois : elle a contribué fortement à l'approvisionnement alimentaire d'Antananarivo et fourni une activité alternative aux nombreux pluriactifs mis à pied par la fermeture des entreprises et le ralentissement de l'activité de la capitale. L'exploitation a ainsi servi de refuge pendant la crise (N'Dienor et Aubry, 2004).

Pour la ville, si l'agriculture, sert toujours « d'espace libre » pour l'urbanisation (voire, par la briqueterie, de «matière première " pour la construction), elle commence à être considérée aussi à travers d'autres fonctions. Sa fonction alimentaire, au moins sur les produits maraîchers, est de plus en plus prise en considération (par exemple, marché spécialisé à Alasora en 2005). Elle est aussi reconnue comme un des moyens les plus efficaces et les moins coûteux de lutter contre les risques d'inondations en plaine. La fonction de valorisation des déchets urbains met en exergue de nouvelles relations ville-agriculture, qui restent largement à créer. Enfin, le retour planifié à la construction sur les hauteurs préserve les zones rizicoles de plaine, au moins autant en raison de leur rôle de régulation des flux hydrauliques que de leur fonction productive directe.

Si nous avons pu vérifier nos hypothèses quant à la différenciation des formes d'agriculture selon l'accès à l'eau et l'accessibilité de la ville, nous confirmons aussi l'hypothèse selon laquelle la durabilité et l'intérêt de la pérennisation in situ de l'agriculture urbaine varient selon les sites et les fonctions remplies. Trois cas sont ainsi rencontrés :

1. des formes d'agriculture a priori durables, car viables économiquement (demandées par le marché), ne gaspillant pas les ressources (entretenant la fertilité des sols) et non condamnées, voire encouragées par les projets urbains. C'est le cas du maraîchage périurbain, en progression, qui peut aussi devenir un lieu d'absorption des déchets urbains ;

2. a contrario, des situations agricoles condamnées par les projets urbains : extension urbaine prévue sur une partie au moins de la plaine sud, poursuivant une dynamique largement entamée (remblais, zones industrielles, « squatterisation » dans ces zones basses). Sur le plan opérationnel, reste à savoir comment accompagner les agriculteurs encore concernés (délocalisations, reconversions?) et, sur le plan scientifique, reste à traiter le problème des répercussions possibles de cette 
urbanisation sur le fonctionnement hydraulique de l'aval de la plaine encore rizicole ;

3. une gamme de situations intermédiaires parmi lesquelles on trouve d'abord les bas-fonds intra-muros, où l'agriculture est dynamique sur le plan économique, a une fonction d'assainissement pour la ville, mais présente des risques sanitaires dans ses produits, voire se heurte à l'urbanisation de fait ou aux projets urbains (tunnel, route). Le dilemme est aigu, car ces zones présentent de forts risques à l'urbanisation (érosion, inondations). Le maintien d'une agriculture (ou d'un autre espace ouvert) suppose une prise de décision des autorités contre l'extension de l'habitat et la résolution des problèmes d'épuration, au moins partielle, des eaux usées. Dans les rizières de la plaine prévues pour être conservées, le problème de durabilité de l'agriculture est endogène (faibles performances techniques et économiques, destruction irréversible du sol par la briqueterie) et exogène (mitage de l'espace par des projets d'infrastructures, répercussions hydrauliques de l'urbanisation d'amont).

Ces premières conclusions n'ont pu être acquises qu'en mobilisant ensemble et en dialogue avec les décideurs les disciplines représentées dans ADURAA. Cependant, des limites apparaissent qui justifieraient des approfondissements disciplinaires et le recours à de nouvelles constructions de projets interdisciplinaires.

La qualité des produits d'agriculture urbaine est largement questionnée, au moins dans certains lieux de production : aborder les répercussions sanitaires de ces conditions de production d'aliments nécessite le recours aux sciences de la nutrition et de la santé. La segmentation éventuelle de filières de produits par la qualité reste aussi une question entière, tant dans sa réalité actuelle que dans ses possibles développements : comment gérer la qualité sanitaire et la garantir dans un État qui a peu de moyens de contrôle ou de sanctions et dont les habitants et consommateurs ont un pouvoir d'achat variable mais souvent faible? L'économie de la qualité dispose ici de cas d'étude innovants. De même, des filières émergentes, techniquement prometteuses et politiquement porteuses, comme la valorisation agricole des déchets urbains, font à leur tour surgir des interrogations : quels éventuels nouveaux risques sanitaires liés à ces déchets? Quelles perceptions éventuellement négatives par les consommateurs des produits utilisant ces déchets urbains?... L'apport de la sociologie et de l'anthropologie est alors important pour étudier risques et perceptions, car ces considérations pourraient remettre en cause ces modes innovants de production. Les conclusions portées à l'issue de ce projet sur la durabilité variable de l'agriculture selon les systèmes et les sites doivent ainsi laisser place à une certaine vigilance et compter sur de nouveaux apports disciplinaires pour être mieux établies.
L'importance croissante de l'agriculture urbaine, corrélative de l'urbanisation mondiale, fait que les questions que nous avons traitées ou que nous nous posons aujourd'hui quant à la durabilité de l'agriculture urbaine à Antananarivo se retrouvent largement ailleurs dans les pays en développement (Bakker et al., 2000 ; Deelstra et Girardet, 2000 ; Smith Olanrewaju et al., 2004). C'est notamment le cas pour la question des risques environnementaux et sanitaires (Mougeot, 2000 ; Armar-Klemesu, 2000 ; Marshall et Te Lintelo, 2001) : la construction méthodologique ici proposée autour de l'instruction pluridisciplinaire des conditions de durabilité pourrait être mise à l'épreuve dans d'autres situations.

\section{Références}

Andriamalala, M., 2006. Relations entre l'urbanisation et l'agriculture dans les bas-fonds intra-muros d'Antananarivo : l'occupation de l'espace et la maîtrise des risques. Thèse de doctorat en géographie, Université d'Antananarivo, Madagascar.

Armar-Klemesu, M., 2000. Urban agriculture and food security, nutrition and health, in Bakker, N., et al. (Eds), Growing Cities, Growing Foods: Urban agriculture on the Policy Agenda, Feldafing, DSE [Fondation allemande pour le développement international], 99-117.

Bakker, N., Dübbeling, M., Gündel, S., Sabel-Koschella, U., de Zeeuw, H., 2000. Growing Cities, Growing Food: Urban Agriculture on the Policy Agenda, Feldafing, DSE.

Bryant, C., 1997. L'agriculture périurbaine : l'économie politique d'un espace innovateur, Cahiers Agricultures, 6, 2, 125-130.

Bryant, C.R., Johnston, T.R.R., 1992. Agriculture in the City's Countryside, London, Belhaven Press.

Charvet, J.-P., 1994. Les agricultures périurbaines. Nouvelles approches et nouvelles questions à propos des agricultures périurbaines, Bulletin de l'Association des géographes français, 2, 119-122.

Cities Alliance (Büschenschütz, M., Oliva Lily, R., Ramiaramanana, J.), 2004. Gestion de l'assainissement liquide et des déchets. Rapport final, in Stratégie de développement de l'agglomération d'Antananarivo, Projet Cities Alliance, CUA-FIFTAMA, Antananarivo.

Commission mondiale sur l'environnement et le développement (CMED), 1987. Notre avenir à tous, New York, Nations unies.

Cour J.-M., 2004. Peuplement, urbanisation et transformation de l'agriculture : un cadre d'analyse demo-économique et spatial, in L'Alimentation des villes, Cah. Agric. Fr., 13, 1, 158-165.

Dabat, M.H., Razafimandimby, S., Bouteau, B., 2004. Atouts et perspectives de la riziculture péri-urbaine à Antananarivo, Madagascar, Cahiers Agricultures, 13, 1, 99-109.

Deelstra,T., Girardet, H., 2000. Urban agriculture and sustainable cities, in Bakker, N., et al. (Eds), Growing Cities, Growing Foods: Urban agriculture on the Policy Agenda, Feldafing, DSE, 43-66.

Donadieu, P., Fleury, A., 2003. La construction contemporaine de la ville-campagne, Revue de géographie alpine, 91, 4, 19-30.

Egziabher, A.G., Lee-Smith, D., Maxwell, D.G., Memon, P.A., Mougeot, L.J.A., Sawio, C.J., 1994. Cities Feeding People: An Examination of Urban Agriculture in East Africa, Ottawa, CRDI. 
Fleury, A., 2005. L'agriculture dans la planification de l'Ile-deFrance : du vide urbain à la multifonctionnalité territoriale, Cahiers de la multifonctionnalité, 8, 33-46.

Godard, O., Hubert, B., 2002. Le Développement durable et la recherche scientifique à l'INRA : rapport à madame la Directrice générale de l'INRA. Rapport intermédiaire de mission, 23 décembre 2002, INRA, Paris.

Jollivet, M., Pavé, A., 1993. L'environnement : un champ de recherche en formation, Natures Sciences Sociétés, 1, 1, 6-19.

Laurent, C., Chevallier, C., Jullian, P., Langlet, A., Maigrot, J.-L., Ponchelet, D., 1994. Ménages, activité agricole et utilisation du territoire : du local au global à travers les RGA, Cahiers Agricultures, 3, 2, 93-107.

Losch, B., 2002. La multifonctionnalité face aux défis de l'agriculture des Suds : une perspective de refondation des politiques publiques? Communication au colloque SFER Multifonctionnalités de l'activité agricole et sa reconnaissance par les politiques publiques, Paris, INA P-G, 21-22 mars.

Marshall, F., Te Lintelo, D., 2001. Pollution effects on urban and periurban agriculture in India: social and economic implications, Urban Agriculture Magazine, 5, 25-26.

Monédiaire, G., 1999. Agricultures urbaines et ville durable européenne: droits et politiques du jardinage familial urbain en Europe, Limoges, Pulim.

Mougeot, L.J.A.. 1995. Faire campagne en ville : l'agriculture périurbaine en Afrique de l'Est, Ottawa, CRDI.

Mougeot, L.J.A., 2000. Urban Agriculture: definitions, presence, potentials and risks, in Bakker, N., et al. (Eds), Growing Cities, Growing Foods: Urban agriculture on the Policy Agenda, Feldafing, DSE, 1-42.

Mougeot, L.J.A., 2005. Agropolis: The Social, Political and Environmental Dimensions of Urban Agriculture, Ottawa, IDRC/ London, Eratscan.

Moustier, P., Mbaye, A., 1999. Introduction, in Moustier, P., et al. (Eds), Agriculture périurbaine en Afrique subsaharienne: actes de l'atelier international du 20 au 24 avril 1998, Montpellier, France, Montpellier, CIRAD, 7-16.

N’Dienor M., 2006. Fertilité et gestion de la fertilisation dans les systèmes maraîchers périurbains des pays en développement: intérêts et limites de la valorisation agricole des déchets urbains dans ces systèmes, cas de l'agglomération d'Antananarivo. Thèse de doctorat, INA P-G, Paris.

N’Dienor, M., Aubry, C., 2004. Diversité et flexibilité des systèmes de production maraîchers dans l'agglomération

Reçu le 6 novembre 2006. Accepté le 8 novembre 2007.
d'Antananarivo (Madagascar) : atouts et contraintes de la proximité urbaine, Cahiers Agricultures, 13, 1, 50-57.

N'Dienor, M., Dabat, M.H., Ramananarivo, R., Randriamiharisoa, F., Rajoelison, J., Aubry, C., 2006. A trend towards urban integration and organization of the tomato subsector in Antananarivo, Madagascar, in Batt, P.J. (Ed.), Proceeding of the First International Symposium Improving the Performance of Supply Chains in the Transitional Economies, ISHS, Chiang Mai, Thailand, July 19-23 2005, Acta Horticulturae, 699.

Nasr, J., Padilla, M. (Eds), 2004. Interfaces : agricultures et villes à l'Est et au Sud de la Méditerranée, Paris, Delta.

Pierrat, A., 2006. La Gestion des déchets à Tananarive : étude de la valorisation des déchets urbains en produits fertilisants. Approche géographique. Mémoire de Master 1 en géographie, Université Paris 1 Sorbonne, Institut de géographie, Paris.

Rahamefy, L., Ramamonjisoa, J., Aubry, C., 2005. L'agglomération d'Antananarivo : projets d'urbanisme et fonctions de l'agriculture, in Multifonctionnalité de l'agriculture dans les territoires périurbains (compte rendu sur CD-ROM de l'atelier "Urbanisme, paysagisme et agriculture », Paris, ENS, 16 décembre 2004), Cahiers de la Multifonctionnalité, 8.

Ramamonjisoa, J., Aubry, C., Dabat, M.H., Andriarimalala M., 2007. Systèmes d'activités en zones agricoles périurbaines à Madagascar. Diversité et flexibilité des exploitations agricoles, in Gafsi, M., Brossier, J., Dugué, P., Jamin, J.-Y., Les Exploitations agricoles familiales en Afrique, Paris, L'Harmattan, Chap. 11, 185-194.

Sachs, I., Weber, J., 1994. Environnement, développement, marché : pour une économie anthropologique, Natures Sciences Sociétés, 2, 3, 258-265.

Smit, J., Nasr, J., 1992. Urban agriculture for sustainable cities: using wastes and idle land and water bodies as resources, Environment and Urbanization, 4, 141-152.

Smit, J., Ratta, A., Nasr, J., 1996. Urban Agriculture: Food, Jobs, and Sustainable Cities, New York, UNDP, Chapter 8, 197-209.

Smith Olanrewaju, B., Moustier, P, Mougeot, L.J.A., Fall, A., 2004. Développement durable de l'agriculture urbaine en Afrique francophone : enjeux, concepts et méthodes, Paris, CIRAD/ Ottawa, CRDI.

Snrech, S., 1997. Croissance démographique et développement urbain: impact sur l'offre et la demande alimentaire, Rome, FAO.

Temple, L., Moustier, P., 2004. Les fonctions et contraintes de l'agriculture périurbaine de quelques villes africaines (Yaoundé, Cotonou, Dakar), Cahiers Agricultures, 13, 1, 15-22. 